\title{
Call for Papers: \\ Priorities in Sexual and Gender Minority Youth Health Research
}

LGBT Health is the premier peer-reviewed journal dedicated to promoting optimal health and health care for all sexual and gender minority (SGM) persons worldwide. The Journal focuses specifically on health while maintaining sufficient breadth to address policy, legislative, educational, and training issues relevant to health care provision and health outcomes. The Journal aims to advance an intersectional understanding of the health needs particular to each SGM population and to improve access to and delivery of affirming clinically and culturally competent health care.

We are seeking novel, high-quality original research articles and systematic reviews and meta-analyses that focus on priorities in SGM youth health research, including the following:

- Identifying points of intervention in the pathways through which minority stress impacts depression, anxiety, and suicidal ideation and behaviors

- Provision of comprehensive affirming care for transgender and non-binary youth

- Reproduction options, decision-making, and outcomes for transgender and nonbinary youth and young adults

- Eating-related pathology among SGM youth

- Effects of violence/trauma on mental and physical health

- Impact of family- and school-based interventions on health and wellbeing

- Strategies to prevent and reduce substance use and misuse

- Physical and mental health of children of SGM parents

- Reducing HIV and other STI risks among SGM youth

- Methodological and ethical considerations in conducting research with SGM youth

- Innovative approaches to minority stress theory including the study of resilience and vulnerability factors and their underpinnings

- Impact of policies and services on SGM youth health

- Health care for SGM youth living out of home (foster care, juvenile justice system, and homeless)

Priority will be given to articles that advance research in these areas and have implications for reducing health disparities and improving clinical practice, access to care, and health outcomes. We welcome articles that address SGM health in the U.S. and all other regions of the world.

All additional information for submission can be found in our Instructions for Authors.

Our mission statement, publications to date, and instructions for authors can be found on our website. We look forward to your submissions.

\section{SUBMIT TODAY}

